\title{
Scenario Planning and Foresight: Advancing Theory and Improving Practice
}

\author{
George Wright, Frances O’Brien, Maureen Meadows, Efstathios Tapinos, and \\ Neil Pyper
}

\begin{abstract}
In this Introduction, we review the logic that underpinned the earlier call for papers and provide a structured sequence for the contents of the sixteen papers that comprise the special issue. Use of particular foresight tools can have predictable effects on strategy making - providing positive changes in mental models and challenging business-as-usual approaches but, more negatively, can also serve to narrow and shape managers' anticipations of the future. Any scenario activity necessarily involves simplification and evaluation processes in knowledge elicitation that need to be carefully monitored for effectiveness. In addition, historical analysis of the focal industry's use of "recipes" can give the scenario practitioner insights into the nature of unfolding futures. Inside the scenario development process, verbal and visual analysis of face-to-face interactions within the scenario team can give insights into process issues and difficulties, and an organisation's prior involvement with scenario activity may give insights into the likely time-demands of any planned activities. Additionally, stakeholders from different organisations can be facilitated to work effectively together in foresight activities. Such facilitated activities can be used to both develop policy and achieve commonly-held objectives. Two papers provide new guidance on the form of successful Delphi applications. Finally, typologies of both foresight methods/approaches and of intervention practices will allow the reflective practitioner to more fully appreciate the characteristics - both positive and negative - of a particular method and/or intervention type.
\end{abstract}

\subsection{Introduction}

The call for papers for this special issue was underpinned by the conference on "Scenario Planning ad Foresight: Advancing Theory and Improving Practice" held at Warwick Business School, Coventry, UK, in December 2018. At that conference, the second in a continuing series, about 70 papers were presented and some were submitted for consideration for inclusion in this special issue. Other papers were submitted for consideration in response to a call for papers that appeared in TFSC at around the same time. We invited papers that focused on scenario and foresight methods in their widest sense that documented the current status of application and use, and that analysed future potential and prospects. Specifically, we invited papers that considered scenario and foresight methods with a focus such as:

* Critical theoretical considerations of a method and its rationale

* Review of the use of a technique in specific applied areas, including evidence of impact on decision making and policy making

This is an accepted author manuscript of the following in-press article: Wright, G., O'Brien, F., Meadows, M., Tapinos, E., \& Pyper, N. (Accepted/In press - 2020). Scenario planning and foresight: advancing theory and improving practice.

Technological Forecasting and Social Change. 
* Analysis and critical evaluation of variations in applications of a method in different contexts - for example, moving beyond the typical application of the scenario method in exploring the external environment for large corporations to applications that encompass scenario planning for, say, governments, industries, or smaller-scale organizations

* Empirical studies comparing method variants - for example, comparing some variant of scenario method with alternative approaches (e.g., forecasting)

* Novel elaborations of a method and critical appraisal of these - for example combinations with the Delphi technique and combinations with inputs from social media

* Consideration of future prospects for a technique

Papers presented at the Warwick conference encompassed this broad field but the papers that appear in this special issue are less broad-ranging and, essentially, deal with five major topic areas: Challenging mental models; Understanding and improving the scenario development process; Enhancing stakeholder engagement in scenario development; Understanding and improving the Delphi process; Developing typologies of foresight activities to improve practice. Details of the constituent papers follow.

\subsection{Challenging and changing mental models}

Frith and Tapinos analyse scenario practice and focus on how particular features of practice lead to particular outcomes of the scenario process. By decomposing descriptions of scenario planning methods, and utilising their own practice-based insights, they identify both contextual factors and process-based cognitive "mechanisms" that demonstrate how scenario planning interventions affect/change participants' mental models - so providing guidance such that subsequent researchers can better provide an evidence-base for the relative efficacy of particular practice components in underpinning particular (positive) outcomes of a scenario-based intervention. As such, the form of future interventions can be designed a-priori.

Burt and Nair focus on the links between organisational learning and strategic foresight. In a 24month longitudinal study of an organisation that had been utilising scenario methods, they demonstrate "unlearning"- defined as letting go of the rigidities of previously held assumptions and beliefs about the contextual environment. They show that the scenario planning process created time and space for the firm's management to understand the limitations of their own preconceptualisations and prior experiences and so opened up new business opportunities - by promoting higher-level intuitions about the business environment.

Onya and Mackay focus on the use of horizon scanning and scenarios in strategy development. In a three-year longitudinal study within a financial services organisation, they argue that the choice of the particular foresight tools that were utilised acted, in itself, to shape and direct the activity of strategy-making by the strategists - thus actively creating a new social reality by either constraining or facilitating opportunities for subsequent action, so-called "performativity". Their study details how horizon-scanning identified trends that "will" (p22) have significant implications for both how the company should market its products and for which customers the company would seek to attract in the future. In addition, use of the horizon scanning approach enrolled input from diverse participants and thus allowed marginal groups to exert more influence on centrally-based strategy making. Use made of horizon-scanning-based presentations within the organisation was shown to be 
persuasive in the development of strategic actions by the organisation - such that "in using the scan... the (strategy) process became more like the world within the scan" (p40).

In summary, use of particular foresight tools can have predictable effects on strategy making providing positive changes in mental models and challenging business-as-usual approaches but, more negatively, can also serve to narrow and shape managers' anticipations of the future.

\subsection{Understanding and improving the scenario development process}

Metz and Hartley analyse scenario development practice and view this activity as, in part, a "valuation process" - since the process includes subjective evaluations such as categorisations and rankings of participant-generated inputs. For example, valuation occurs when uncertainties and the impacts of these uncertainties - when they are resolved as outcomes- are assigned weights or values on the Intuitive logic's impact/predictability matrix. Metz and Hartley's "valuation lens" is reflexive and allows us to understand how practice as information in the process is summarised and simplified - with the result that meaning is created and shared between participants.

Ram focuses on one aspect of evaluation, that of option evaluation against constructed scenarios using multi-criterion methods, to rate the relative quality of alternative options. Using a simulation approach, she analyses the "robustness" of options against the range of constructed scenarios and provides a quantitative appreciation of how the "utility loss" of a choice option is influenced both by the extant scenarios as well as the overall choice criterion. She concludes that the decision maker should take into account both his/her attitude to risk and the nature of the scenario set under consideration - since the latter may, or may not, indicate the vulnerability of the choice options.

Steinmann, Auping and Kwakkel introduce "scenario discovery" as a means of supporting decision making by reducing complexity due to deep uncertainty. Scenario discovery analyses the results of computational experiments that map out the consequences of uncertainties, jointly. In their paper, the authors develop behavior-based scenario discovery to allow analysis of dynamic changes in outcomes - by integrating time-series-based clustering of computational experiments.

Bezjian, Stoyanova, McKiernan and MacKay focus on "industry recipes" and how knowledge of these can enhance scenario development when there is known to be competitive rivalry within a particular industry - documented by analysing the plights of both challenger and incumbent organisations. Using an in-depth case study of the Hollywood film industry, these authors demonstrate how our appreciation of causality can be enhanced by an understanding of how industry recipes both constrain and facilitate the unfolding of particular scenario storylines. For example, taken-forgranted work practices and historical utilisation of particular technologies may be part of a particular firm's future trajectory. Using an interview-based approach, Bezjian et al examine historical change and its causes within the Hollywood film industry. They conclude that the impacts of emerging driving forces on current industry recipes can be better understood and then used to enhance scenario development.

Meadows and O'Brien analyse audio and video data from a strategy workshop to show how scenario tools are utilised in practice. Focusing on "micro-activities", they analyse conversational discourse and social interactions and show that visual content analysis can enhance conversational analysis. The scenario activity utilised pre-developed scenarios within a strategy-making context over a 24hour period. The authors observed an iterative process - iterating between idea sharing, working with post-its and flip charts, and reflecting on the validity of the strategy proposals that emerged 
from participants. They noted that the received scenarios provoked technical debate about their use in strategy development, content debate, and, also, efforts to develop social cohesion and agreement within the scenario team.

Ramirez, Bhatti and Tapinos investigate whether scenario development becomes quicker after repetitive application within an organisation. Using a questionnaire-based approach, these authors found that the actual time spent on a scenario planning activity was generally lower than anticipated and that the more scenario planning an organisation has undertaken then the less time a fresh activity actually takes. Counter-intuitively, those organisations that spend more time on scenario planning than anticipated were smaller than those who spent less time, suggesting that, in large organisations, experience is more important in estimating time commitment.

In summary, any scenario activity necessarily involves simplification and evaluation processes in knowledge elicitation that need to be carefully monitored for effectiveness. In addition, historical analysis of the focal industry's use of "recipes" can give the scenario practitioner insights into the nature of unfolding futures. Within the scenario development process, verbal and visual analysis of face-to-face interactions within the scenario team can give insights into process issues and difficulties. Finally, an organisation's prior involvement with scenario activity may give insights into the likely time-demands of any planned activities.

\subsection{Enhancing stakeholder engagement in scenario development}

Gattringer and Wiener focus on "interorganisational foresight", where several organisations agree to collaborate in foresight activities. They discuss issues to do with openness, knowledge-sharing and trust in the start-up phase of any collaboration. They note that multi-agency activity may not be about the development of a common perspective but instead it may focus on the elucidation of variety in perspectives on the future. Using an action research approach, they document three such foresight activities and conclude that predictors of a successful outcome are: enthusiasm for a crossindustry exchange of viewpoints; early-stage interaction between participants; clarity in individuallevel benefits to the multi-organisation activity; and professional management of the overall activity. They conclude that the individual-level goals of participant organisations in any such activity should be complementary, if not fully aligned with one-another.

Lehoux, Miller and Williams-Jones focus on the design of public engagement methods to inform "anticipatory governance" - for situations in which technological innovations present future societal opportunities and problems. With foresight, such issues can be anticipated, such that appropriate policy and legal frameworks can be developed a-priori. These authors discuss how participation can be facilitated to articulate what the public believes should, or should not, happen in the future - so that ethical dilemmas are resolved early on. In their empirical study, participant reactions to technology-based scenarios were elicited, such that moral deliberations about the contents of the scenario could be captured - so-called "prospective deliberation". This focus on "moral imagination" in the prospective deliberations allows public engagement to be enacted and then utilised by policymakers in their anticipatory governance.

Gordon analyses the scenario process that produced the "Mont Fleur" scenarios at the point of the transition to black majority rule in South Africa. He shows how the emergence of a "normative" desired future scenario to actually become the external reality was enabled by the deliberate construction of the scenario-building team - both in terms of representative stakeholder diversity and the current (and future) access-to-power of the team membership. In this way, Gordon argues, a 
desired future was facilitated - aided by achieved political reconciliation founded on the identification of a common goal - the desired future of the nation - within the group of powerholding stakeholders.

In summary, stakeholders from different organisations can be facilitated to work effectively together in foresight activities. In addition, such facilitated activities can be used to both develop policy and achieve commonly-held objectives.

\subsection{Understanding and improving the Delphi process}

Belton, MacDonald, Wright, and Hamlin focus on improving one foresight method, Delphi, by developing a six-step "prescription" for the design of well-founded and defensible Delphi applications. They include discussion of issues to do with: choice of respondents; survey question design; software delivery choice; feedback choices; respondent drop-out; and analysis and presentation of the Delphi yield. At each step, there are choice options and Belton et al discuss the pros and cons of each option.

Kattirtzi and Winskel, more narrowly, focus on the use of Delphi for policy making on the future of energy systems as the United Kingdom responds to the challenge of extensive decarbonisation. Policy Delphi focuses on documenting and understanding similarities and differences between expert viewpoints - based on differences in information sources, uncertainties in available evidence, or fundamental value differences. But, the latter class of viewpoint differences are potentially irreconcilable - even with further search for information or evidence. In their empirical work using Delphi, these authors elicited numerical estimates from participants, together with underpinning rationales and reasoning. These authors argue that sources of information should be elucidated within these rationales, where possible, so it becomes clear to the Delphi administrator whether a disagreement amongst Delphi participants could, potentially, be resolved by further information - or could not because of underlying differences in value.

In summary, the two papers in this section provide new guidance on the form of successful Delphi applications.

6.0 Developing typologies of foresight activities to improve practice

Minkkinen, Auffermann and Ahokas develop a typology of six "foresight frames" that underpin any foresight activity by focussing participants' attention on what is deemed to be relevant and important to the foresight activity manager - the two major dimensions of relevance being "level of perceived unpredictability" and "level of pursued change". The former is underpinned by an assumption of closed versus open causal systems of interacting environmental driving forces, and the latter by the desire to achieve a particular future as opposed to simply understanding the nature of possible futures. They conclude that each of the different foresight approaches contains different assumptions that, once appreciated, allow the defensible selection of a particular foresight approach and, in addition, allows the design of multimethod activities.

Crawford focuses on scenario-based interventions in organisations. She notes the variety of "schools" or approaches and argues for the need for a typology to guide best-practice interventions that allows comparisons to be made between intervention types. In this way, facilitators of 
interventions can both evaluate and improve particular methods. By applying the attributes of her intervention typology to a? previously-published case, she identifies its particular strengths and weaknesses in terms of: projects goals; the design of the intervention process; the content of the developed scenarios; and the impact of the process/content on decision makers and their subsequent decision making.

In summary, these typologies of both foresight methods/approaches and of intervention practices will allow the reflective practitioner to more fully appreciate the characteristics - both positive and negative - of a particular method and/or intervention type.

\subsection{Conclusion}

This special issue has brought together fifteen papers as a collection that captures the state-of-theart of scenario planning and foresight related research at this moment in time. The papers cover a broad range of topics - addressing theoretical developments in the field and providing insights for improved practice. This special issue is the third in a series in this journal. Comparing the clustering of the papers across the three special issues (Wright et al., 2013; Wright et al., 2018) we can observe that there is a continuity in the study of method. The field seems to have reached a consensus on the basic elements of the scenario planning method and now the focus has shifted in exploring how to achieve specific outcomes, like valuation and evaluation, or how to improve the application of the method with wider engagement of stakeholders. At the same time, it is evident that the latest publications in the general field of anticipation are theorising practices, in order to interpret and explain the empirical observations of issues in practical applications. Overall, we observe that authors are looking to different fields of social science in order to broaden the debate and apply new lens' of interpretation. Finally, in this latest special issue, we note new attempts to improve the overall practice of foresight independent of the particular method used. The direction and growth of the field of anticipation is a testament of its evolving maturity over a number of years. Looking forward, we call for further research that continues to balance the advancement of theory with the improvement of practice.

\section{References}

Include the details of the sixteen papers listed under the heading - Article collections: Scenario planning and foresight: advancing theory and improving practice

Wright, G., Cairns, G. and Bradfield, R. Scenario methodology: new developments in theory and practice. Technological Forecasting and Social Change, 2013, 80, 561-565

Wright, G., Meadows, M., Tapinos, S., O'Brien, F. and Pyper, P. Improving scenario methodology: theory and practice. Introduction to the special issue. Technological Forecasting and Social Change, 2017, 124, 1-5 